\title{
Transverse coherent transition radiation for diagnosis of modulated proton bunches
}

\author{
A. Pukhov and T. Tueckmantel \\ Institut fuer Theoretische Physik I, Universitaet Duesseldorf, 40225 Duesseldorf, Germany
}

(Received 21 May 2012; published 5 November 2012)

\begin{abstract}
Transverse coherent transition radiation (TCTR) emitted by a relativistic particle bunch traversing a conducting surface is analyzed. The bunch emits dipolelike radiation in the direction transverse to the bunch axis when the beam radius is smaller than the radiation wavelength. The radiation wavelength is defined by the longitudinal structure of the particle bunch. The particular case of proton bunches modified by propagation in plasma, but still carrying an unmodulated current, is considered. Radius-modulated bunches with a constant current emit axially symmetric radiation. Hosed bunches emit antisymmetric radiation in the plane of hosing. The TCTR field amplitude may reach $100 \mathrm{kV} / \mathrm{m}$ for the existing proton bunches.
\end{abstract}

Coherent transition radiation (CTR) is one of the most common techniques used for diagnosis of a longitudinal structure of charged particles bunches [1-5]. The method particularly demonstrated its power to characterize accelerated electron bunches in laser-plasma experiments [6-8]. An elementary charge propagating through a medium with a particular dielectric permittivity is dressed by a field matched to that medium. When the charge traverses a sharp boundary of two media with different permittivities, its field must be adjusted. The unmatched field can be radiated. The strongest radiation is observed when a charge passes a boundary between a conductor and vacuum. A pointlike relativistic charge with the relativistic factor $\gamma$ emits a radiation burst that is collimated within a cone with the opening angle $\theta \approx 1 / \gamma$ around the axis, although the emission is exactly zero in the propagation direction itself. The radiation is broadband. A bunch of particles can emit this radiation coherently at the wavelength comparable with its longitudinal structure.

Recently, a concept of proton bunch-driven plasma wakefield accelerator has been put forward [9-11]. In this concept, a long proton bunch is sent through plasma where it undergoes self-modulation at the plasma wave period and excites a strong resonant wakefield. A test experiment is in preparation at CERN. One of the experimental challenges will be the detection and characterization of the proton bunch modulation after it exits the plasma cell.

The nature of the proton bunch modulation is such that the proton bunch radius is modulated, but the total bunch current remains the same in each cross section. For this reason, there will be no signatures of the proton bunch modulation in the forward coherent transition radiation.

Published by the American Physical Society under the terms of the Creative Commons Attribution 3.0 License. Further distribution of this work must maintain attribution to the author(s) and the published article's title, journal citation, and DOI.
The classic forward CTR is useless in this case. Moreover, it is important in the experiment to distinguish between the axisymmetric modulation mode when the radius of the proton bunch is changing periodically [11] and the possible hosing mode when the proton bunch centroid oscillates periodically in the transverse plain [12].

Below we show that the transverse coherent transition radiation (TCTR) does contain the signature of the bunch modulation and allows one to distinguish between the axisymmetric modulation mode and the hosing. The TCTR is emitted perpendicularly to the particle bunch propagation direction and its amplitude does not depend on the particle's $\gamma$ factor as soon as it is large enough.

\section{ORIGIN OF TRANSVERSE TRANSITION RADIATION}

Let us consider a transition radiation emitted by a particle bunch as it traverses normally a boundary between a conductor plate and vacuum. The interaction geometry is illustrated in Fig. 1. When an elementary charge $d q$ exits from the conducting plate in the normal direction with the velocity $\mathbf{v}$, the radiated field is given by the LienardWiechert formula [13]:

$$
\begin{aligned}
\mathbf{d E} & =\frac{d q}{c^{2}\left(R-\frac{\mathbf{R} \cdot \mathbf{v}}{c}\right)^{3}} \mathbf{R} \times\left[\left(\mathbf{R}-\frac{\mathbf{v}}{c} R\right) \times \frac{d \mathbf{v}}{d t^{\prime}}\right] \\
& +\frac{d q}{c^{2}\left(R+\frac{\mathbf{R} \cdot \mathbf{v}}{c}\right)^{3}} \mathbf{R} \times\left[\left(\mathbf{R}+\frac{\mathbf{v}}{c} R\right) \times \frac{d \mathbf{v}}{d t^{\prime}}\right],
\end{aligned}
$$

where $t^{\prime}$ is the retarded time so that

$$
t^{\prime}+R\left(t^{\prime}\right) / c=t .
$$

The second term in Eq. (1) is generated by the image of the physical charge in the conducting plate.

When the elementary charge is inside the metal plate, its field is completely screened. Thus, the current is created abruptly when the charge exits into the free space. We can 


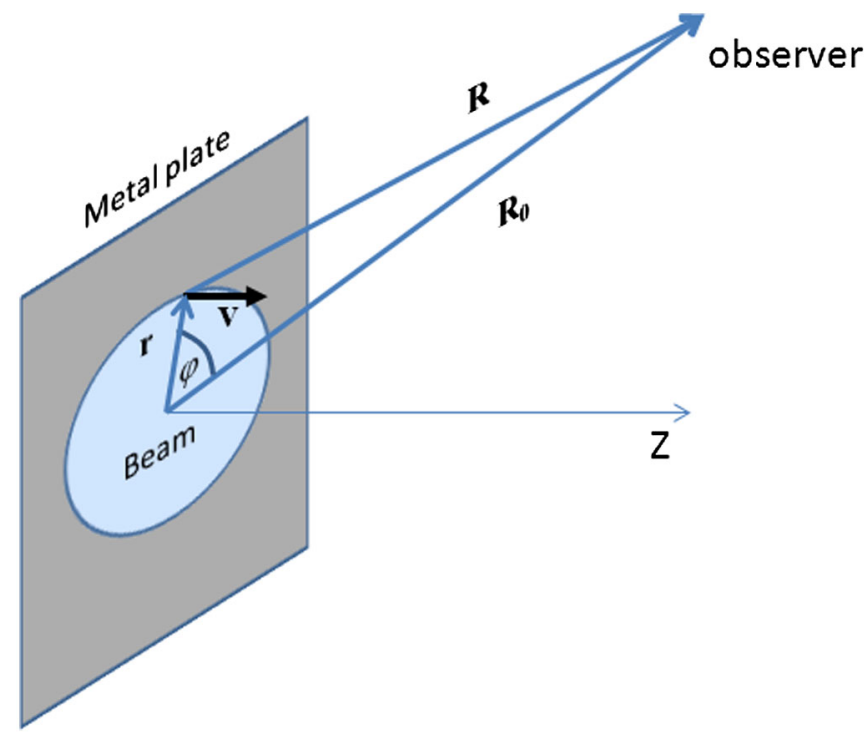

FIG. 1. Geometry of the transverse coherent transition radiation. A charged particle bunch exits a conductor plate normally to the surface. The observer looks at the emitted radiation from side to the beam, in the plane of the surface. The vector $\mathbf{R}_{0}$ points from the bunch axis to the observer.

write for the velocity $\mathbf{v}\left(t^{\prime}\right)=\mathbf{v}_{0} \Theta\left(t^{\prime}-t_{\mathrm{e}}^{\prime}\right)$, where $t_{\mathrm{e}}^{\prime}$ is the time the charge exits into vacuum and $\Theta(t)$ is the Heaviside step function.

The denominators in Eq. (1) suggest that a pointlike charge emits the strongest field at the angle $\theta \approx 1 / \gamma$ around the propagation direction. Yet, we will not be interested in the emission in this direction, because it does not contain any coherent information about the bunch modulation. Rather, we are looking in the transverse plane, along the surface of the conducting plate. It is the dipolelike radiation of the particle bunch.

For the radiation emitted in the transverse plane, Eq. (1) becomes especially simple, because $\mathbf{v} \perp \mathbf{R}$ :

$$
\mathbf{d E}=-\frac{2 \mathbf{v}_{0} d q}{c^{2} R} \delta\left(t^{\prime}-t_{e}^{\prime}\right)
$$

This is essentially the dipole radiation formula. For a continuous bunch, $d q=e n(t, \mathbf{r}) v_{0} d S d t$, where $d S=$ $r d r d \varphi$ is the area element in the bunch cross section and $n$ is the local particle density. The field is polarized in the bunch propagation direction and normal to the metal plate surface. The observed radiated field is thus

$$
E\left(t, R_{0}\right)=-\frac{2 e v_{0}^{2}}{c^{2} R_{0}} \iint n\left(t^{\prime}, \mathbf{r}\right) d S,
$$

where we have assumed the observer being far away from the source and set $R \approx R_{0}$ in the denominator. At the same time, the retarded time is

$$
t^{\prime}=t-\frac{R\left(t^{\prime}\right)}{c} \approx t-\frac{R_{0}}{c}+\frac{r}{c} \cos \varphi .
$$

Now we are ready to apply the formula (4) to specific particle bunches. We are interested primarily in proton bunches that have undergone self-modulation or hosing after they propagated in plasma. The peculiarity of these proton bunches is that the current is not modulated. Only the bunch radius may be modulated or the centroid position is changing (hosing). We show that self-modulation and hosing result in TCTR with different signatures that can be experimentally observed.

\section{TCTR EMITTED BY MODULATED AXIALLY SYMMETRIC BUNCHES WITH CONSTANT CURRENT}

In this section we consider TCTR of a axially symmetric particle bunch whose radius is modulated. For simplicity, we assume the Gaussian transverse bunch density distribution,

$$
n\left(t^{\prime}, \mathbf{r}, z\right)=n_{0} \frac{\sigma_{0}^{2}}{\sigma^{2}\left(t^{\prime}, z\right)} e^{-r^{2} / 2 \sigma^{2}\left(t^{\prime}, z\right)} .
$$

The total current carried by the bunch of the profile (6) is constant in any cross section: $J=2 \pi \sigma_{0}^{2} e n_{0} v_{0}$.

Let us assume the bunch radius is modulated so that

$$
\begin{aligned}
\sigma\left(t^{\prime}, z\right) & =\sigma_{0}\left[1+\epsilon f\left(k z-k v_{0} t^{\prime}\right)\right] \\
& =\sigma_{0}\left[1+\epsilon f\left(k z-k v_{0} t+k R_{0} \frac{v_{0}}{c}-k r \frac{v_{0}}{c} \cos \varphi\right)\right],
\end{aligned}
$$

and the modulation depth is small, $\epsilon \ll 1$. Further, we assume that the particle bunch is narrow, so that $k \sigma_{0} \ll 1$. We Taylor expand (6), keep terms linear in $\epsilon$ and quadratic in $k r$, and set the metal plate position at $z=-R_{0} v_{0} / c$ :

$$
n(t, \mathbf{r}, z) \approx n_{0}\left[1+\epsilon A\left(\frac{r^{2}}{\sigma_{0}^{2}}-2\right)\right] e^{-r^{2} / 2 \sigma_{0}^{2}},
$$

where

$$
\begin{aligned}
A= & f\left(-k v_{0} t\right)-k r \frac{v_{0}}{c} f^{\prime}\left(-k v_{0} t\right) \cos \varphi \\
& +\frac{1}{2}\left(\frac{k r v_{0}}{c}\right)^{2} f^{\prime \prime}\left(-k v_{0} t\right) \cos ^{2} \varphi
\end{aligned}
$$

The second and the third terms in (9) originate from the time retardation (2) and are essential for the radiation emission.

The field excited by the axisymmetric particle bunch is then

$$
\begin{aligned}
E\left(t, R_{0}\right)= & -n_{0} \frac{2 e v_{0}^{2}}{c^{2} R_{0}} \int_{0}^{2 \pi} d \varphi \\
& \times \int_{0}^{\infty} r\left[1+\epsilon A\left(\frac{r^{2}}{\sigma_{0}^{2}}-2\right)\right] e^{-r^{2} / 2 \sigma_{0}^{2}} d r .
\end{aligned}
$$

The integrand in (10) contains terms with different powers of $k r$. The terms independent on $k r$ are not retarded. The oscillatory part of the integral of these terms vanishes 
because we assumed the total current of the particle bunch being constant. These terms describe radiation at the wavelength defined by the bunch current envelope. The terms linear in $k r$ contain the factor $\cos \varphi$ and disappear when we integrate in the angle $\varphi$ over the bunch cross section. This is due to the axial symmetry of current distribution in the bunch. The first oscillatory terms that do not vanish after the integration are quadratic in $k r$. Collecting the terms proportional to $(k r)^{2}$ at the first time harmonic, we get

$$
\begin{aligned}
E\left(t, R_{0}\right)= & \epsilon n_{0} f^{\prime \prime}\left(k v_{0} t\right) \frac{2 e v_{0}^{4}}{R_{0} c^{4}} \int_{0}^{2 \pi} \cos ^{2} \varphi d \varphi \int_{0}^{\infty} r(k r)^{2} \\
& \times\left(1-\frac{r^{2}}{2 \sigma_{0}^{2}}\right) e^{-\left(r^{2} / 2 \sigma_{0}^{2}\right)} d r .
\end{aligned}
$$

Integrating (11) we find the expression for the radiation field at the distance $R_{0}$ from the beam axis:

$$
E\left(t, R_{0}\right)=-2 \epsilon \frac{J}{c R_{0}}\left(k \sigma_{0}\right)^{2} \beta_{0}^{3} f^{\prime \prime}\left(-k v_{0} t\right) .
$$

The field is proportional to the radius modulation amplitude $\epsilon$, square of the bunch radius $\left(k \sigma_{0}\right)^{2}$, and the second derivative of the modulation function $f^{\prime \prime}\left(\omega_{\mathrm{rad}} t\right)$.

In the case of a periodic harmonic modulation of the particle bunch radius, $f\left(k v_{0} t\right)=\cos \left(k v_{0} t\right)$, the expression (12) becomes

$$
E\left(t, R_{0}\right)=2 \epsilon \frac{J}{c R_{0}}\left(k \sigma_{0}\right)^{2} \beta_{0}^{3} \cos \omega_{\mathrm{rad}} t,
$$

where $J=2 \pi \sigma_{0}^{2} e n_{0} v_{0}$ is the total bunch current, $\beta_{0}=$ $v_{0} / c$ and the emitted radiation frequency is

$$
\omega_{\text {rad }}=k v_{0} .
$$

We remember that the expression (12) is valid only for narrow bunches, $k \sigma_{0} \ll 1$. Wide particle bunches, $k \sigma_{0} \gg 1$, do not radiate coherently in the transverse direction. The coherent radiated field becomes exponentially small for large bunch radii. Thus, the optimal condition for TCTR is $k \sigma_{0} \sim 1$. This case is hard to treat analytically and we have to rely on numerical simulations then. Still, the expression (12) gives us a good guide to estimate the TCTR of an axially symmetric proton bunch whose radius is modulated, but the total current in each cross section remains constant.

As the proton beam in our case is modulated in plasma with some particular density $n_{e}$, the modulation wave number $k$ is given by the plasma wave number $k_{p}=$ $\omega_{p} / c$, where $\omega_{p}=\sqrt{4 \pi n_{e} e^{2} / m_{e}}$ is the plasma frequency. One can normalize the field (13) on the so-called plasma wave breaking field $E_{W B}=m_{e} c \omega_{p} / e$ :

$$
\frac{E\left(t, R_{0}\right)}{E_{W B}}=\frac{2 \epsilon}{k R_{0}} \frac{J}{J_{A}}\left(k \sigma_{0}\right)^{2} \beta_{0}^{3} \cos \omega_{\mathrm{rad}} t,
$$

where $J_{A}=m c^{3} / e=17 \mathrm{kA}$ is the natural current value. The plasma wave breaking field is given by the engineering formula $E_{W B} \approx \sqrt{n_{e} / 10^{14} \mathrm{~cm}^{-3}} \mathrm{GV} / \mathrm{m}$. Although the formula (15) has been derived for narrow weakly modulated bunches, it shows that the radiated field strength may reach huge values for the available proton bunches when these are deeply modulated, $\epsilon \sim 1$, and their radius is comparable with the modulation wavelength, $k \sigma_{0} \sim 1$.

\section{TCTR EMITTED BY HOSED BUNCHES WITH CONSTANT CURRENT}

Now we consider TCTR of a particle bunch whose radius is constant, while the centroid position oscillates periodically in a plane. We assume the Gaussian transverse bunch density distribution,

$$
n\left(t^{\prime}, \mathbf{r}, z\right)=n_{0} e^{-\left[\mathbf{r}-\mathbf{r}_{c}\left(t^{\prime}, z\right)\right]^{2} / 2 \sigma^{2}}
$$

Again, the bunch is assumed to be thin, $k \sigma \ll 1$. The total current carried by the bunch of the profile (16) is constant in any cross section: $J=2 \pi \sigma^{2} e n_{0} v_{0}$.

For the bunch centroid displacement we write

$$
\begin{aligned}
\mathbf{r}_{c}\left(t^{\prime}, z\right) & =\mathbf{r}_{c 0} h\left(k z-k v_{0} t^{\prime}\right) \\
& =\mathbf{r}_{c 0} h\left(k z-k v_{0} t+k R_{0} v_{0} / c-k r\left(v_{0} / c\right) \cos \varphi\right),
\end{aligned}
$$

where the bunch centroid displacement is small, $\left|\mathbf{r}_{c 0}\right| / \sigma \ll 1$. We Taylor expand (16), keep terms linear in $k r$, and set the metal plate position at $z=-R_{0} v_{0} / c$ :

$$
n(t, \mathbf{r}, z) \approx n_{0}\left(1+\frac{\mathbf{r}_{c 0} \mathbf{r}}{\sigma^{2}} A_{h}\right) e^{-r^{2} / 2 \sigma_{0}^{2}}
$$

where

$$
A_{h}=h\left(-k v_{0} t\right)-k r \frac{v_{0}}{c} h^{\prime}\left(-k v_{0}\right) t \cos \varphi
$$

and

$$
\mathbf{r}_{c 0} \mathbf{r}=r_{c 0} r \cos \left(\varphi-\varphi_{0}\right)
$$

with $\varphi_{0}$ being the angle between the plane of hosing and the direction to the observer.

Because the hosed bunch is not axisymmetric, it is sufficient to keep the linear terms in $k r$ only. The second term in (19) originates again from the time retardation (2) and it is this term that leads to the radiation emission.

The field excited by the axisymmetric particle bunch is then

$$
\begin{aligned}
& E\left(t, R_{0}\right) \\
& =-n_{0} \frac{2 e v_{0}^{2}}{c^{2} R_{0}} \int_{0}^{2 \pi} d \varphi \int_{0}^{\infty} r\left(1+\frac{r_{c 0} r \cos \left(\varphi-\varphi_{0}\right)}{\sigma^{2}} A_{h}\right) \\
& \quad \times e^{-\left(r^{2} / 2 \sigma_{0}^{2}\right)} d r .
\end{aligned}
$$

In the integrand in (21), we collect terms linear in $k r$. The terms independent on $k r$ are not retarded. The oscillatory part of the integral of these terms vanishes because we assumed the total current of the particle bunch being 
constant. The terms, linear in $k r$, contain the factor $\cos \varphi$ and do not disappear in general. Collecting the terms proportional to $k r$ and integrating, we get

$$
\begin{aligned}
E\left(t, R_{0}\right) & =2 \pi \cos \varphi_{0} h^{\prime}\left(-k v_{0} t\right) \frac{e n_{0} v_{0}^{3}}{R_{0} c^{3}} \sigma^{2} k r_{c 0} \\
& =\frac{J}{c R_{0}} \beta_{0}^{2} k r_{c 0} \cos \varphi_{0} h^{\prime}\left(-k v_{0} t\right) .
\end{aligned}
$$

The radiation amplitude is proportional to the hosing amplitude $k r_{0}$ and the first derivative of the hosing function $h^{\prime}\left(\omega_{\text {rad }} t\right)$. In the case of a periodic harmonic hosing of the particle bunch radius, $h(k z)=\cos (k z)$, the expression (22) becomes

$$
E\left(t, R_{0}\right)=\frac{J}{c R_{0}} \beta_{0}^{2} k r_{c 0} \cos \varphi_{0} \sin \left(k v_{0} t\right) .
$$

From (22) and (23) we see that the hosed bunch radiation is not axially symmetric. The main lobes are located in the hosing plane as described by the factor $\cos \varphi_{0}$ and are antisymmetric to each other.

Again, one can normalize the field (23) on the plasma wave breaking field $E_{W B}=m_{e} c \omega_{p} / e$ if the proton bunch has been hosed during its propagation in plasma:

$$
\frac{E\left(t, R_{0}\right)}{E_{W B}}=\frac{1}{k R_{0}} \frac{J}{J_{A}} \beta_{0}^{2} k r_{c 0} \cos \varphi_{0} \sin \left(k v_{0} t\right) .
$$

The radiated field (24) scales linearly with the hosing amplitude $k r_{0}$.

\section{NUMERICAL EXAMPLES}

We performed two test numerical simulations to illustrate the effect of TCTR. The simulations have been done with the hybrid code H-VLPL3D [14,15]. The code can handle plasmas of arbitrary high densities as fluids. In these test simulations, we take a model proton bunch that carries the current of an optimized super-proton-synchrotron (SPS) bunch, $I_{0}=50 \mathrm{~A}$ and the protons have the longitudinal momentum $p=450 \mathrm{GeV} / \mathrm{c}$. The length of the model bunch was chosen short, $\sigma_{z}=2.4 \mathrm{~mm}$, for illustration purposes. The final result does not depend on the bunch length.

In the first simulation of a modulated proton bunch, we set up the model density profile,

$$
n(t, \mathbf{r}, z)=n_{0} \frac{\sigma_{0}^{2}}{\sigma^{2}\left(t^{\prime}, z\right)} e^{-r^{2} / 2 \sigma^{2}(t, z)} e^{-z^{2} / 2 \sigma_{z}^{2}},
$$

with $n_{0}=1.4 \times 10^{12} \mathrm{~cm}^{-3}$ and the unperturbed transverse radius $\sigma_{0}=0.2 \mathrm{~mm}$. The actual bunch radius is modulated as $\sigma=\sigma_{0}\left(1+0.5 \cos k_{p} z\right)$, where $k_{p}=$ $2 \pi / \lambda_{p}$ is the plasma wave number. For the plasma density of $n_{e}=7 \times 10^{14} \mathrm{~cm}^{-3}$ we have the plasma wavelength $\lambda_{p}=1.2 \mathrm{~mm}$.

In the simulation, we send the proton bunch through a high density plasma slab that would represent a conducting metal foil. The foil, the proton bunch, and the radiated field are

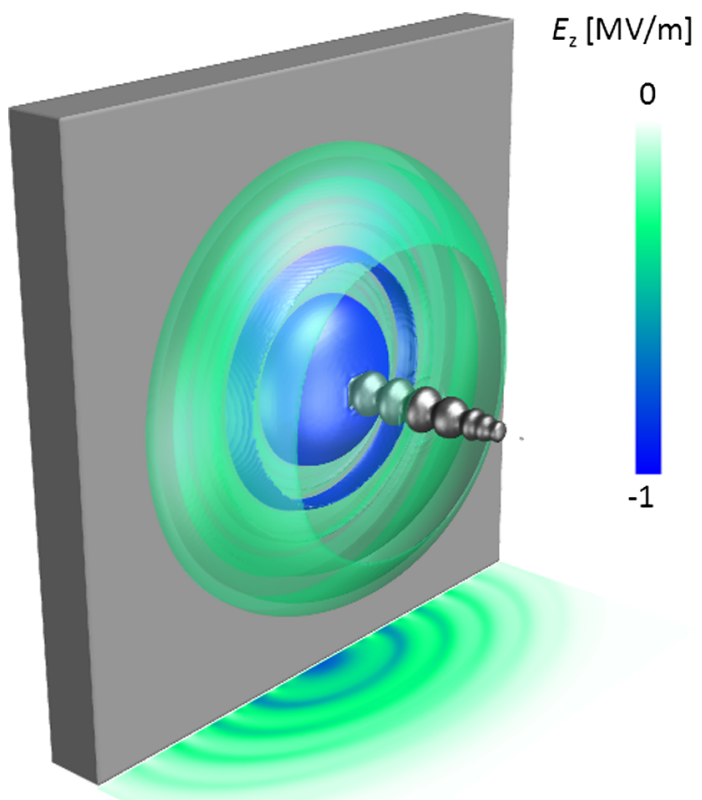

FIG. 2. TCTR emitted by a proton bunch exiting from a metallic foil. Only the radius of the proton bunch is modulated, not the current. The colored surfaces show the electric field component normal to the metal foil.

shown in Fig. 2. The radiation is emitted axisymmetrically in the transverse direction. The radiation field magnitude reaches several $100 \mathrm{kV} / \mathrm{m}$ values at $1 \mathrm{~mm}$ distance from the bunch axis.

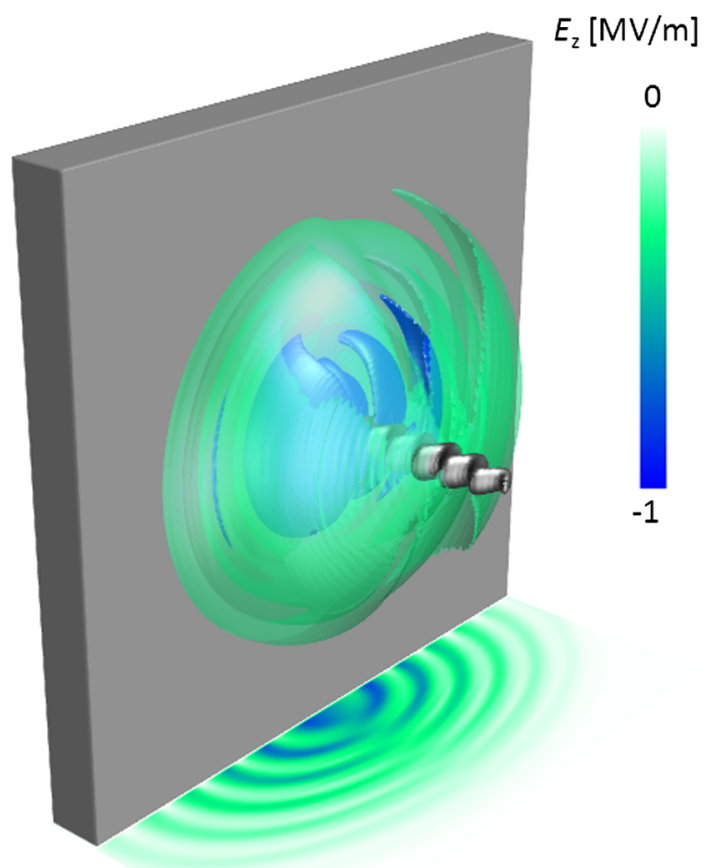

FIG. 3. TCTR emitted by a hosed proton bunch exiting from a metallic foil. The radiated field is shaken off antisymmetrically in the plane of hosing. 
In the second simulation we used a hosed proton bunch with the model density profile,

$$
n(t, \mathbf{r}, z)=n_{0} e^{-\left(x-x_{0}(t, z)\right)^{2} / 2 \sigma_{0}^{2}} e^{-z^{2} / 2 \sigma_{z}^{2}},
$$

with $n_{0}=1.4 \times 10^{12} \mathrm{~cm}^{-3}, \sigma_{0}=0.2 \mathrm{~mm}$, and the time dependent centroid $x_{0}=r_{0} \cos k_{p} z$, where the hosing amplitude was $r_{0}=0.2 \mathrm{~mm}$. The radiation produced by this proton bunch as it traverses a metal foil is shown in Fig. 3. The radiation is no more axisymmetric. Rather, the hosed bunch "shakes off" its field in the direction of hosing. The radiation field again reaches $100 \mathrm{kV} / \mathrm{m}$ values at $1 \mathrm{~mm}$ distance from the bunch axis.

\section{CONCLUSIONS}

It has been shown that a particle bunch that is modulated in radius or hosed does radiate TCTR even when the total current of the bunch remains unmodulated. The radiation is emitted due to the retardation effect: the observer located in the transverse plane sees signals of particles traversing the conductor plate at retarded times. The integral of this retarded current does show a time dependence at the bunch modulation period. An axisymmetric particle bunch with a periodically modulated radius emits axisymmetric waves. On the contrary, a hosed bunch whose centroid oscillates in a transverse plane emits in a direction perpendicular to this plane and does not emit in the plane of hosing. In both cases, the field of the coherent radiation scales linearly with the bunch current and is proportional to the modulation amplitude. The TCTR is emitted only when the bunch radius is smaller than or comparable with the radiation wavelength. The emission is maximized when the bunch radius is $k \sigma \sim 1$, because in this case the retardation effects maximize while the coherence is not yet affected by the finite bunch radius. The numerical simulations suggest that plasma-modulated SPS proton bunches may emit TCTR fields at the characteristic amplitudes of $100 \mathrm{kV} / \mathrm{m}$.

\section{ACKNOWLEDGMENTS}

This work was supported by Deutsche Forschungsgemeinschaft.

[1] J. Rosenzweig, G. Travish, and A. Tremaine, Nucl. Instrum. Methods Phys. Res., Sect. A 365, 255 (1995).

[2] U. Happek, A. J. Sievers, and E. B. Blum, Phys. Rev. Lett. 67, 2962 (1991).

[3] H.-C. Lihn, P. Kung, C. Settakorn, and H. Weidemann, Phys. Rev. E 53, 6413 (1996).

[4] R. Lai and A. J. Sievers, Phys. Rev. E 52, 4576 (1995).

[5] Y. Shibata, T. Takahashi, T. Kanai, K. Ishi, and M. Ikezawa, Phys. Rev. E 50, 1479 (1994).

[6] W. P. Leemans, C. G. R. Geddes, J. Faure, Cs. T'oth, J. van Tilborg, C. B. Schroeder, E. Esarey, G. Fubiani, D. Auerbach, B. Marcelis et al., Phys. Rev. Lett. 91, 074802 (2003).

[7] C.B. Schroeder, E. Esarey, J. van Tilborg, and W.P. Leemans, Phys. Rev. E 69, 016501 (2004).

[8] J. van Tilborg, C. B. Schroeder, C. V. Filip, Cs. Toth, C. G. R. Geddes, G. Fubiani, R. Huber, R. A. Kaindl, E. Esarey, and W. P. Leemans, Phys. Rev. Lett. 96, 014801 (2006).

[9] A. Caldwell, K. Lotov, A. Pukhov, and F. Simon, Nat. Phys. 5, 363 (2009).

[10] A. Caldwell, K. Lotov, A. Pukhov, and G. Xia, Plasma Phys. Controlled Fusion 53, 014003 (2011).

[11] A. Pukhov, N. Kumar, T. Tuckmantel, A. Upadhyay, K. Lotov, P. Muggli, V. Khudik, C. Siemon, and G. Shvets, Phys. Rev. Lett. 107, 145003 (2011).

[12] N. Kumar, A. Pukhov, and K. Lotov, Phys. Rev. Lett. 104, 255003 (2010).

[13] L. D. Landau and E. M. Lifshitz, The Classical Theory of Fields, Fourth Edition: Volume 2, Course of Theoretical Physics Series (Butterworth-Heinemann, Washington, DC, 1980).

[14] A. Pukhov, J. Plasma Phys. 61, 425 (1999).

[15] T. Tuckmantel, A. Pukhov, J. Liljo, M. Hochbruck, IEEE Trans. Plasma Sci. 38, 2383 (2010). 